Pacific Journal of Mathematics

MOIRE PHENOMENA IN ALGEBRAIC GEOMETRY: KeITH MiLo Kendo 


\title{
MOIRÉ PHENOMENA IN ALGEBRAIC GEOMETRY: POLYNOMIAL ALTERNATIONS IN $R^{n}$
}

\author{
KeITH M. KendiG
}

This paper introduces an object in algebraic geometry akin to but different from an algebraic variety. The main idea is this: The concept of "inverse image under a polynomial map of a point" (=algebraic variety) is replaced by "inverse image under a polynomial map of a periodic subset." In this paper $R$ is the groundfield, and the periodic subset is taken to be $[0,1)+2 Z \subseteq R$. These inverse images, which we call polynomial alternations, are, in $\boldsymbol{R}^{2}$, like diffraction gratings encountered in optics. They are closed under complementation as well as "mod two sum." This sum is like intersection for ordinary varieties in at least one important way - an analogue of the usual dimension theorem holds under mod two sum. Union and intersection are dual, and each gives rise to a phenomenon not encountered with ordinary varieties - namely striations, or "moire fringes" are formed. These fringes run along algebraic varieties, and these varieties correspond to linear combinations of the polynomials defining the alternations. A density is induced in each algebraic variety, and this natural density is itself periodic. It depends on the coefficients of the linear combination; the author determines this function.

1. Introduction. Suppose that on a transparent sheet one inks in a parallel family of straight bands, to create a "diffraction ruling." Assume that all bands (the inked-in ones as well as the clear ones) have the same width. If another such sheet is placed on the first one, and if the two families are almost (but not exactly) parallel, then there is created a third family of "bands" or "fringes." These fringes will be wider, and more widely-spaced, than the original bands, and almost perpendicular to them. (See Figure 1.) More generally, if the second family of bands differs from the first by a linear transformation $T$ which is close to but not equal to the identity map, then in their union we will see a new set of wider and more widely-spaced fringes, whose orientation depends on $T$. This phenomenon is not restricted to families of straight bands: Figure 2 shows the moire phenomenon arising from the union of two slightly-displaced Fresnel zone plates, and Figure 3, from the union of a straight-line plate with a Fresnel plate.

Moiré phenomena have been noticed in the physical sciences, and have found a number of important applications, particularly in 


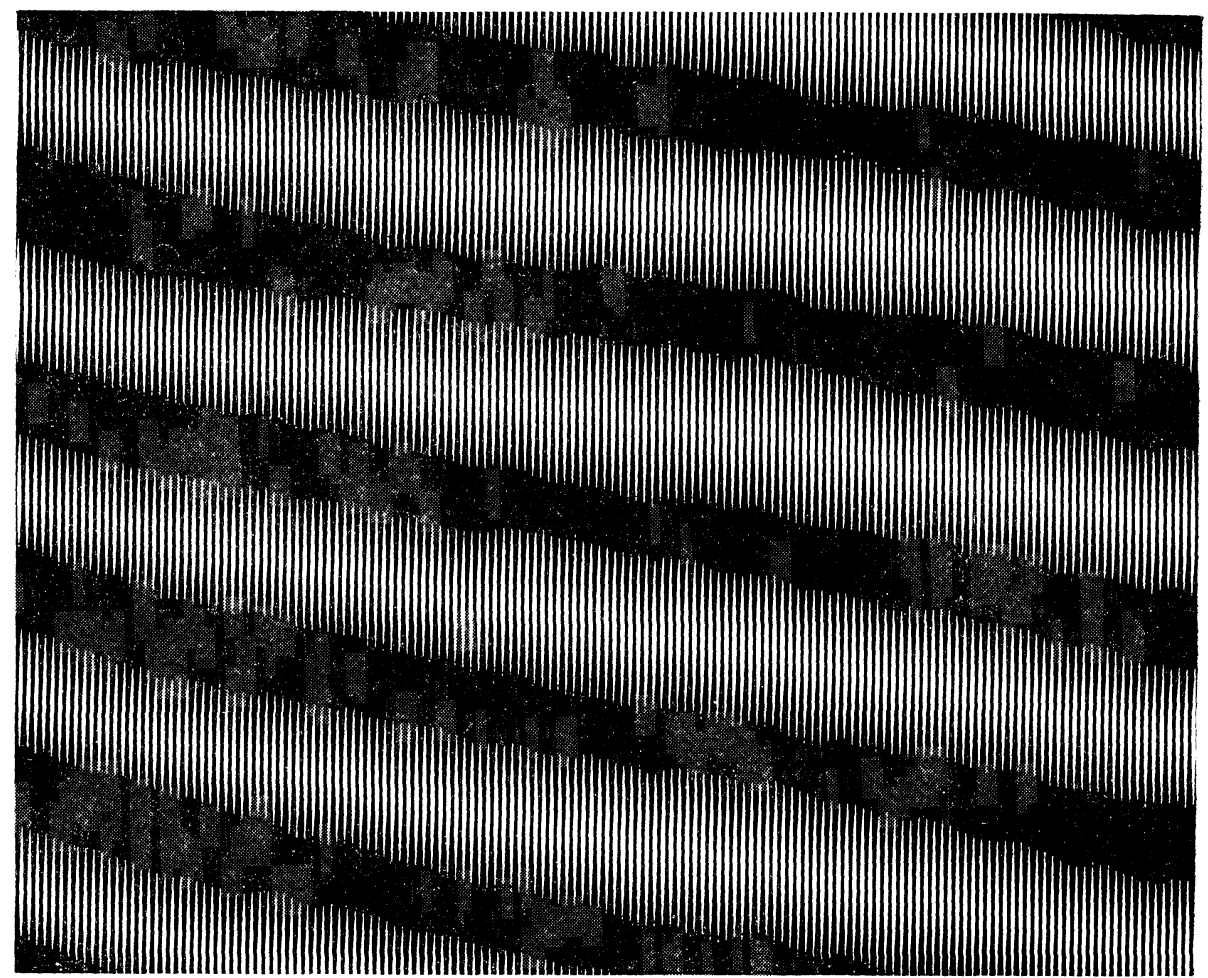

FIGURE 1

stress-strain analysis and crystallography. As far as the author can tell, such phenomena have not been given a general, purely mathematical treatment. We make a beginning in this paper.

2. A linear case in $\boldsymbol{R}^{2}$. In this section we look more carefully at the parallel-band example of $\S 1$. We do three things: first, we determine the direction and spacing of the "primary" or "first-order" fringes; second, we determine the direction and spacing of the socalled "higher-order" fringes; third, we obtain a basic "density function" for fringes of any order. We briefly explain.

The "primary" fringes are the large, obvious ones in Figures 1 and 2. But for example in Figure 3 (the union of a straight-line diffraction ruling and a Fresnel zone plate), we see not only a prominent family of fringes, but also "higher-order" families of circles, centered further and further away from the center of the picture. This phenomenon, which we explain more fully later on, is actually typical in moirés. Although these higher-order families are not so evident to the eye in Figures 1 and 2, in Figure 1, for example, the intersection of any line in $\boldsymbol{R}^{2}$ with the pattern in Figure 1, defines on that line a density, which in every case is at 


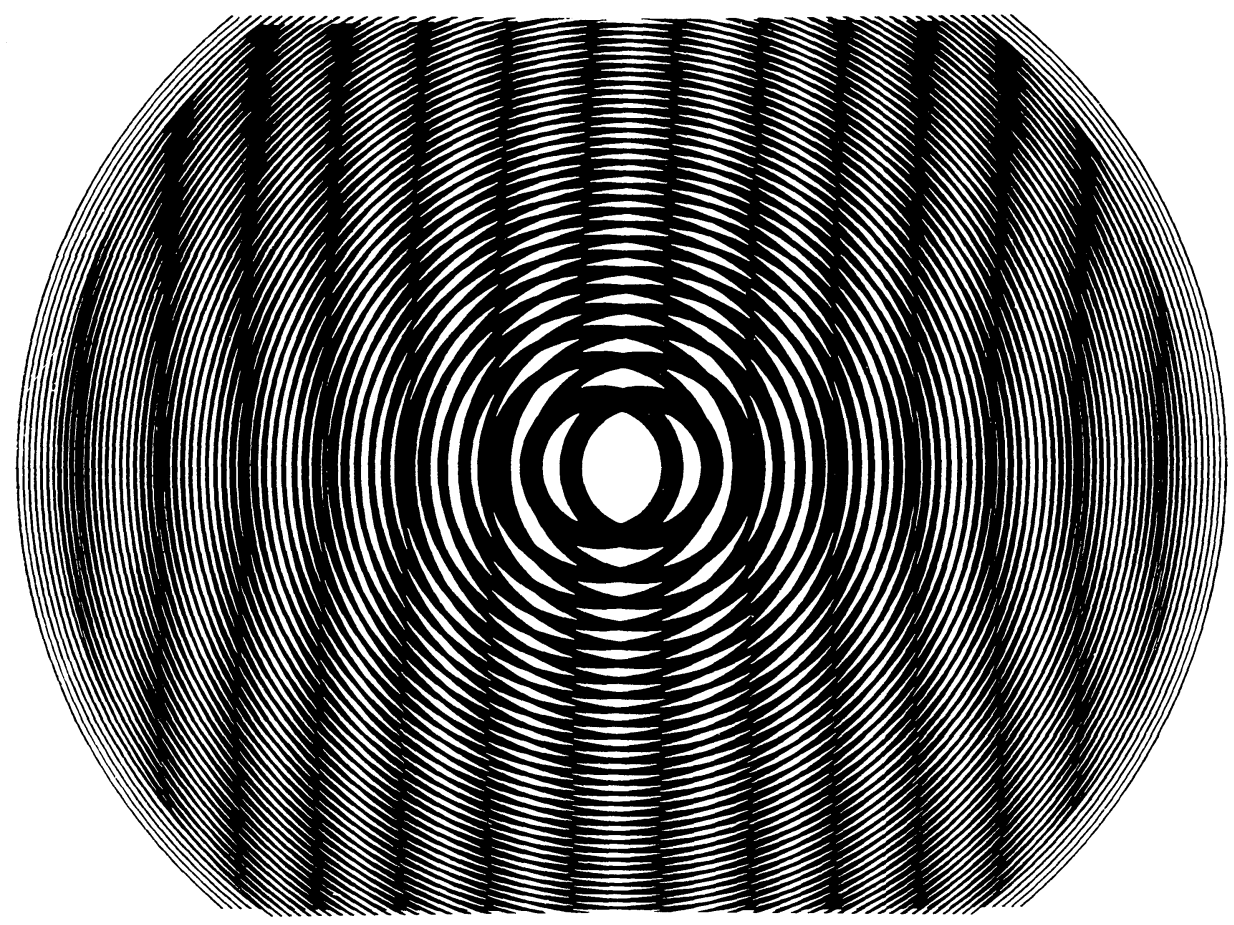

Figure 2

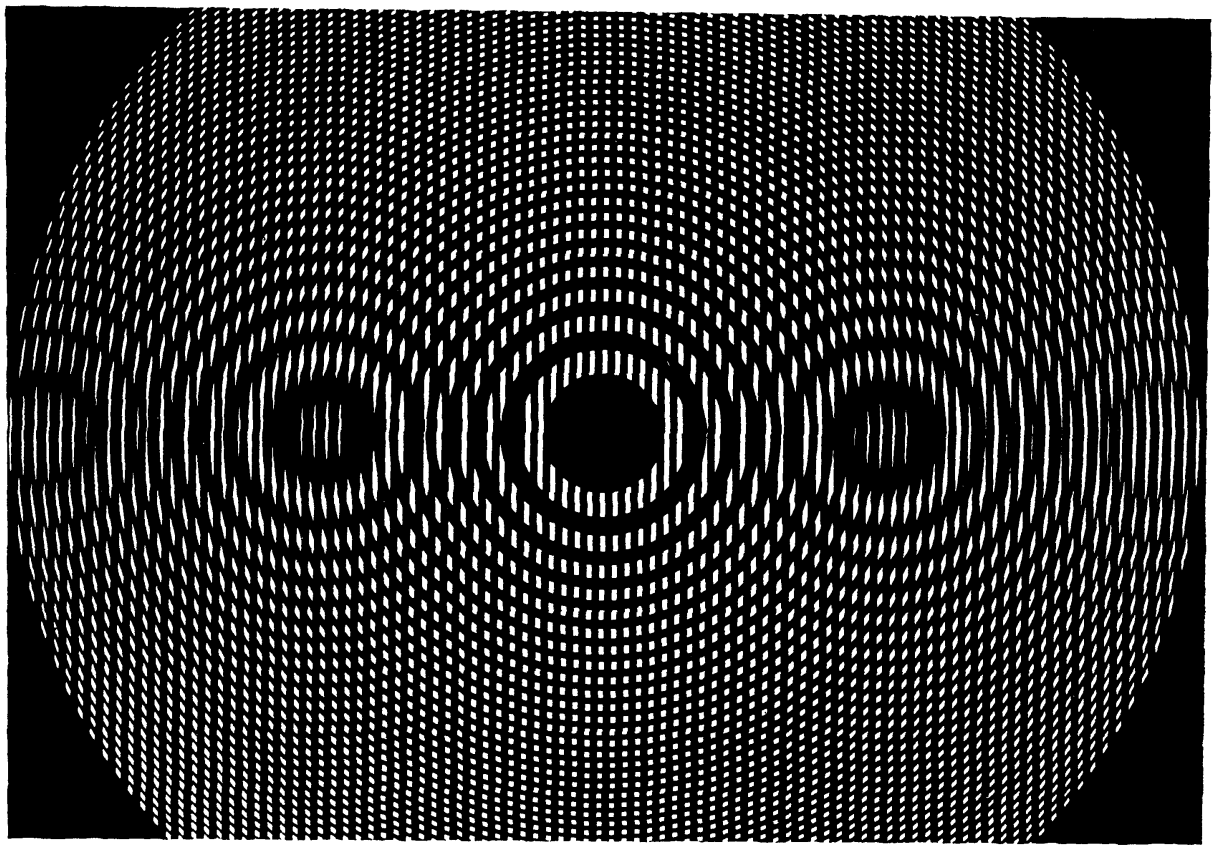


least $1 / 2$. It turns out that a "random" line intersects the pattern in density 3/4. However for many lines, the density is different from $3 / 4$, and as one parallel-translates such a line, the density oscillates between a minimum and a maximum, similar to the way the densities on lines parallel to the primary fringes in Figure 1 oscillate, in an obvious way, between $1 / 2$ and 1 . Later in this paper, we generalize this density function from one-dimensional linear, to an algebraic, arbitrary-dimensional setting (\$6).

We insert here the following definition of density, which will suffice for our immediate purposes. (We generalize Definition 2.1 slightly in Definition 4.2.)

Definition 2.1. Let $S$ be a countable union of intervals in $\boldsymbol{R}$. Then $S$ has density $D$ in $R$ if for any $\varepsilon>0$, there is a decomposition of $\boldsymbol{R}$ into a disjoint union of intervals $T_{\imath} \subseteq \boldsymbol{R}$, which are uniformly bounded in length, so that

$$
\left|\triangleright-\frac{\text { length }\left(S \cap T_{i}\right)}{\text { length } T_{i}}\right|<\varepsilon
$$

for all but possibly finitely many $T_{i}$.

Now, to begin, denote $\left(X_{1}, X_{2}\right)$ by $X$, let $a=\left(a_{1}, a_{2}\right)$ be a nonzero element of the real 2-plane $\boldsymbol{R}_{X_{1} X_{2}}=\boldsymbol{R}_{X}$, and define $\boldsymbol{A}^{1}$ to be the subset $[0,1)+2 \boldsymbol{Z}$ of $\boldsymbol{R}$. ( $\boldsymbol{A}^{1}$ is a special case of $\boldsymbol{A}^{n} \cong \boldsymbol{R}^{n}$ in Definition 4.1.) Then $f(X)=a \cdot X$ maps from $\boldsymbol{R}_{X}$ to $\boldsymbol{R}$, and $f^{-1}\left(\boldsymbol{A}^{1}\right)$ is a parallel family of bands in $\boldsymbol{R}_{X}$. The bands run in a direction orthogonal to $a$, each band has width $1 /|a|\left(=1 / \sqrt{\left.a_{1}^{2}+a_{2}^{2}\right)}\right.$, and each is separated from the next band by a distance of $1 /|a|$. We denote $f^{-1}(\boldsymbol{A})$ by $V^{*}(f)$.

Let $a^{\prime}=\left(a_{1}^{\prime}, a_{2}^{\prime}\right)$ be another nonzero element of $\boldsymbol{R}_{X}$; if $g(X)=$ $a^{\prime} \cdot X$, then $V^{*}(g)=g^{-1}\left(\boldsymbol{A}^{1}\right)$ is also a set of bands on $\boldsymbol{R}_{X}$. Let us assume that $a$ and $a^{\prime}$ are linearly independent, and consider the union $V^{*}(f) \cup V^{*}(g)$. Then

(2.2). The lines $\left(a+a^{\prime}\right) \cdot X=n$ and $\left(a-a^{\prime}\right) \cdot X=n(n \in \boldsymbol{Z})$ are contained in $V^{*}(f) \cup V^{*}(g)$ exactly when $n$ is odd.

It is easily checked that $a+a^{\prime}$ and $a-a^{\prime}$ are the only vectors for which (2.2) holds.

EXAMPLE 2.3. Let $a=(1,0), a^{\prime}=(0,1)$. Then $f(X),=X_{1}, g(X)=$ $X_{2}$, and $V^{*}(f)$ consists of parallel vertical bands. Throughout this paper, it will be convenient to regard, e.g., $f^{-1}([0,1))$ as a "black band," and $f^{-1}([1,2))$ as a "white band." Thus $V^{*}(f)$ consists of alternating black and white bands, each band having width $1 ; V^{*}(g)$ 


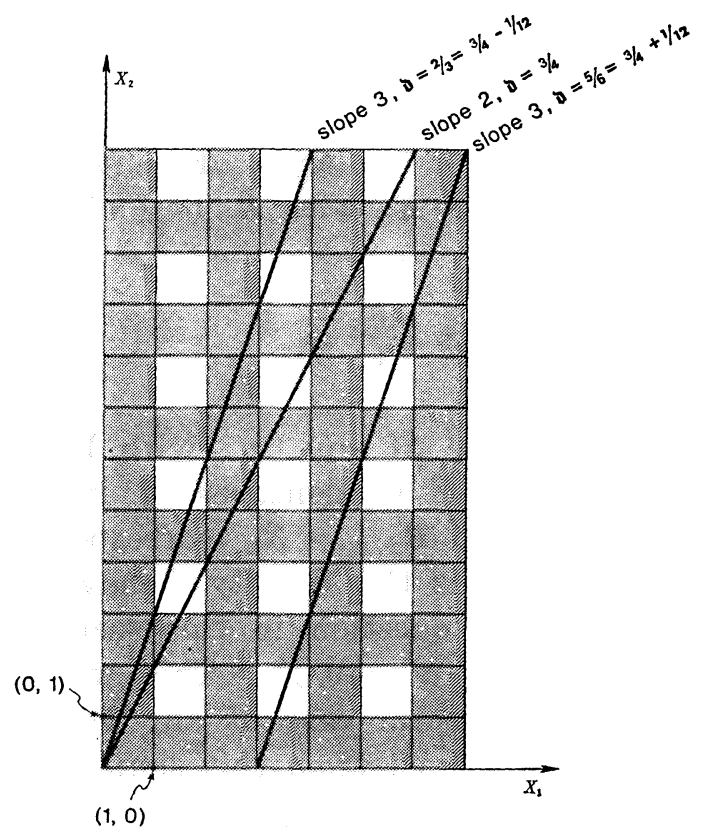

Figure 4

is the same, only horizontal. The lines $X_{2}= \pm X_{1}+n$ ( $n$, an odd integer) are contained in $V^{*}\left(X_{1}\right) \cup V^{*}\left(X_{2}\right)$. (See Figure 4.) Note that as we parallel-translate, e.g., the line $X_{2}=X_{1}$ (or $X_{2}=-X_{1}$ ), the intersection with $V^{*}\left(X_{1}\right) \cup V^{*}\left(X_{2}\right)$ varies in density. For instance, the line $X_{2}=X_{1}$ intersects $V^{*}\left(X_{1}\right) \cup V^{*}\left(X_{2}\right)$ in density $1 / 2$, while the line $X_{2}=X_{1}+1$ intersects it in density 1. For any $b(0 \leqq b \leqq 1)$, the line $L_{b}: X_{2}=X_{1}+b$ intersects $V^{*}\left(X_{1}\right) \cup V^{*}\left(X_{2}\right)$ in density $(1+b) / 2$. The density then decreases linearly from 1 back to $1 / 2$ as $b$ increases from 1 to 2 ; thus the density oscillates in a piecewise linear way between 1 and $1 / 2$. Similarly for lines of slope -1 .

We can express the oscillatory behavior in the above example in a more compact way. First, let us introduce this notation:

Notation 2.4. Let $\Delta(x): \boldsymbol{R} \rightarrow \boldsymbol{R}$ denote the periodic "triangle function" defined in the interval of periodicity $[-1,1)$ by

$$
\Delta(x)=1-2|x| \text {. }
$$

The density $\mathfrak{D}\left(L_{b}\right)$ in which any line $L_{b}: X_{2}= \pm X_{1}+b$ intersects $V^{*}\left(X_{1}\right) \cup V^{*}\left(X_{2}\right)$ is then

$$
\mathfrak{D}\left(L_{b}\right)=\frac{3}{4} \mp \frac{\grave{d}(b)}{4} .
$$

In the general case considered before Example 2.3, the distance 
between two maximum-density (or two minimum-density) lines $\left(a-a^{\prime}\right) \cdot X=n$ and $\left(a-a^{\prime}\right) \cdot X=n+2$, is $2 /\left|a-a^{\prime}\right|$; in this case of $\left(a+a^{\prime}\right) \cdot X=n$ and $\left(a+a^{\prime}\right) \cdot X=n+2$, it is $2 /\left|a+a^{\prime}\right|$. Of course if $a$ is close to $a^{\prime}$, then $2 /\left|a-a^{\prime}\right|$ is large. Finally, the density of intersection of $V^{*}(\alpha \cdot X) \cup V^{*}\left(a^{\prime} \cdot X\right)$ with $L_{b}:\left(\alpha \pm a^{\prime}\right) \cdot X=b$ is easily seen to be

$$
\frac{3}{4} \pm \frac{\Delta(b)}{4}
$$

3. Density of intersection of any line with $V^{*}\left(X_{1}\right) \cup V^{*}\left(X_{2}\right) \subseteq$ $\boldsymbol{R}_{X_{1} x_{2}}$. So far, we have looked at densities of intersection along only two different directions in $\boldsymbol{R}_{X_{1} X_{2}}=\boldsymbol{R}_{X}$ (corresponding to slopes \pm 1 in Example 2.3). What is the density behavior along other directions? We consider this question in this section. As in Example 2.3, we take $a=(1,0), a^{\prime}=(0,1)$. This will simplify considerations; results in the general case of any two linearly independent $a, a^{\prime}$ can easily be derived from this simple case.

Convention 3.1. In this section, $M$ will always mean the "moiré" $V^{*}\left(X_{1}\right) \cup V^{*}\left(X_{2}\right) \subseteq \boldsymbol{R}_{V_{2} X_{2}}$.

We will first consider lines of rational slope. Then we consider lines of irrational slope.

To begin, let $L \subseteq \boldsymbol{R}_{X}$ be a line of slope $m=n_{2} / n_{1}\left(n_{\imath} \in \boldsymbol{Z}\right)$. We can at once write down the density in the degenerate case when $n_{1} n_{2}=0$.

(3.2) If either (but not both) $n_{2}$ or $n_{1}$ is zero (corresponding to $m=0$ or $\infty)$, then the density $\mathfrak{D}\left(L_{b}\right)$ in which $n_{1} X_{2}=n_{2} X_{1}+b$ intersects $M$ is

$$
\begin{aligned}
& \mathfrak{D}\left(L_{b}\right)=1 \text { if } b \in \boldsymbol{A}^{1} ; \\
& \mathfrak{D}\left(L_{b}\right)=\frac{1}{2} \text { if } b \notin \boldsymbol{A}^{1} .
\end{aligned}
$$

In view of (3.2), we now assume that in $m=n_{2} / n_{1}, n_{1}$ and $n_{2}$ are nonzero and relatively prime. We shall further assume that $n_{1}$ is odd, for if $n_{1}$ were even, then $n_{2}$ would be odd, and arguments similar to those given below would work throughout.

We want to get an analogue of (2.4.1) and (2.5). We start by finding the maximum and minimum densities; this amounts to finding the amplitude of the oscillation term. It will then be easy to obtain an appropriate generalization.

We first find the density for a particular $L$ - the one through $(0) \in \boldsymbol{R}_{X}$; we denote this 1 -subspace by $L_{0}$. The density of $M \cap L_{0}$ in $L_{0}$ will turn out to be a minimum. Note that $M \cap L_{0}$ is periodic 
and that, in obvious notation, the half-open line segment $S=[(0,0)$, $\left.\left(2 n_{1}, 2 n_{2}\right)\right)$ is a period. It therefore suffices to find the density in $S$ - i.e., to find length $(M \cap S) /$ length $S$. Also, note that $\boldsymbol{R}_{X}$ is the disjoint union of unit squares, $\boldsymbol{R}_{X}=\bigcup_{i, j \in \boldsymbol{Z}}((i, j)+([0,1) \times[0,1)))$. To find the density, we shall slightly rearrange the intersections of these squares with $M$.

To do this, we note that the segment $S_{1}=\left[(0,0),\left(n_{1}, n_{2}\right)\right)$ lies in the rectangular region $\left[0, n_{1}\right) \times\left[0, n_{2}\right)$; this region is in turn the union of $n_{1}$ smaller rectangular regions

$$
R_{i}=[i-1, i) \times\left[0, n_{2}\right) \quad\left(i=1,2, \cdots, n_{1}\right) .
$$

Similarly, the segment $S_{2}=\left[\left(n_{1}, n_{2}\right),\left(2 n_{1}, 2 n_{2}\right)\right)$ lies in $\left[n_{1}, 2 n_{1}\right) \times\left[n_{2}, 2 n_{2}\right)$ and this last is the union of

$$
R_{i}^{\prime}=\left(n_{1}, n_{2}\right)+R_{\imath} \quad\left(i=1,2, \cdots, n_{1}\right) .
$$

(Note that all the $R_{i}$ and $R_{i}^{\prime}$ are mutually congruent.) Now interchange $M \cap R_{i}$ with $M \cap R_{i}^{\prime}$ for $i=1,3,5, \cdots, n_{1}$. Within the new region $\left[0, n_{1}\right) \times\left[0, n_{2}\right)$, we have an ordinary checkerboard pattern consisting of black and white squares. All four corner squares of the checkerboard are white if $n_{2}$ is odd; if $n_{2}$ is even, two of the corner squares are white and two are black. The new region $\left[n_{1}\right.$, $\left.2 n_{1}\right) \times\left[n_{2}, 2 n_{2}\right)$ is completely black. It is clear that these interchanges do not affect the density of $M \cap S$ in $S$. After this rearrangement, the density in $S_{2}$ is of course 1 . It remains to determine the density in $S_{1}$; we then simply average the two densities.

To find the density in $S_{1}$, let us replace each of the $n_{1} n_{2}$ unit squares in the $n_{1} \times n_{2}$ checkboard, by a finer $n_{2} \times n_{1}$ checkerboard (again white in all four corners if $n_{2}$ is odd, and white in only two corners if $n_{2}$ is even), obtained by dividing the horizontal sides of each unit square into $n_{2}$ equal parts, and the vertical sides into $n_{1}$ equal parts. (The "squares" in this finer checkerboard are actually little $1 / n_{2} \times 1 / n_{1}$ rectangles.) It is easy to see that the intersection of $S_{1}$ with this new "refined" $n_{1} n_{2} \times n_{1} n_{2}$ checkerboard is the same as the intersection of $S_{1}$ with the $n_{1} \times n_{2}$ before refinement. Now consider the set $D$ of all points in $[0,1) \times[0,1)$ which are congruent $\bmod \boldsymbol{Z} \times \boldsymbol{Z}$ to some point in $S_{1}$. $D$ consists of various line segments, all parallel to each other. In fact, $D$ is the union of diagonals - one diagonal from each of the little $1 / n_{2} \times 1 / n_{1}$ rectangles in $[0,1) \times[0$, 1). This last follows from the relative primality of $n_{1}$ and $n_{2}$. (One can also regard $S_{1}$ as winding around the checkerboard torus obtained by reducing the refined checkerboard modulo $\boldsymbol{Z} \times \boldsymbol{Z}$. $S_{1}$ winds around $n_{1}$ times one way, and $n_{2}$ times the other way.) Since the intersection of $S_{1}$ with any such $1 / n_{1} \times 1 / n_{2}$, rectangle is always a diagonal of that rectangle, and since the ratio of diagonal to area 
of any of these $1 / n_{2} \times 1 / n_{1}$ rectangles is constant, the density of $S_{1}$ 's intersection with the refined checkerboard can be found by counting the black $1 / n_{2} \times 1 / n_{1}$ rectangles in $[0,1) \times[0,1)$. If $n_{2}$ is odd, then there are $\left(n_{1} n_{2}-1\right) / 2$ of them, since the four corner rectangles are white. If $n_{2}$ is even, there are $n_{1} n_{2} / 2$ of them. In the first case, the density in $S_{1}$ is $\left(n_{1} n_{2}-1\right) / 2 n_{1} n_{2}$, and in the second case it is $n_{1} n_{2} / 2 n_{1} n_{2}=1 / 2$.

We now average the densities in $S_{1}$ and in $S_{2}$ to conclude that when $n_{2}$ is odd, $L_{0}$ intersects $M$ in density

$$
\grave{D}\left(L_{0}\right)=\frac{1}{2}\left(1+\frac{n_{1} n_{2}-1}{2 n_{1} n_{2}}\right)=\frac{3}{4}-\frac{1}{4 n_{1} n_{2}} ;
$$

when $n_{2}$ is even, $L_{0}$ intersects $M$ in density

$$
\mathfrak{D}\left(L_{0}\right)=\frac{3}{4} .
$$

Now that we have determined the density when $L$ is a subspace $L_{0}$ of rational slope, we next find the density for any line $L_{b}: n_{1} X_{2}=$ $n_{2} X_{1}+b,(b \in \boldsymbol{R})$. We begin with the case when $b=i \in \boldsymbol{Z}$.

For this, consider the parallelogram-shaped region $A \in \boldsymbol{R}_{X}$ which is the image of $I^{2}=[0,1) \times[0,1)$ under the linear transformation sending $(1,0)$ to $\left(n_{1}, n_{2}\right)$ and $(0,1)$ to $\left(0, n_{2}\right)$. There are exactly $n_{1} n_{2}$ (integral) lattice points in $A$. Now each of the $n_{1} n_{2}$ equations

$$
n_{1} X_{2}=n_{2} X_{1}+i \quad\left(i=0,1, \cdots, n_{1} n_{2}-1\right)
$$

has an integer solution. (Since $n_{1}$ and $n_{2}$ are relatively prime, it is clear that for $i=1$, (3.5) has an integer solution $\left(X_{1}, X_{2}\right)=\left(x_{1}, x_{2}\right)$. For arbitrary $i,\left(i x_{1}, i x_{2}\right)$ is a solution.) Also, for any such solution $\left(i x_{1}, i x_{2}\right),\left(i x_{1}+n_{1}, i x_{2}+n_{2}\right)$ is also a solution; it is easy to check that one gets all solutions this way. This implies that for each $i=0,1, \cdots, n_{1} n_{2}-1$, there is exactly one lattice point in $A \cap L_{2}$. Since there are $n_{1} n_{2}$ lines $L_{i}$ defined by the equations in (3.5), we have accounted for all $n_{1} n_{2}$ lattice points in $A$.

We can now determine the density in $L_{\imath}$, as follows. If $i$ is even, then $M \cap L_{i}$ is the same as $M \cap L_{0}$ (up to a parallel-translation), and if $i$ is odd, $M \cap L_{i}$ and $M \cap L_{0}$ are (set-theoretic) complements (up to a parallel-translation). In the first case, $\mathrm{D}\left(L_{i}\right)=3 / 4-$ $1 / 4 n_{1} n_{2}$; in the second case, the term $\left(n_{1} n_{2}-1\right) / 2 n_{1} n_{2}$ in (3.3) is of course replaced by $1-\left(n_{1} n_{2}-1\right) / 2 n_{1} n_{2}$; averaging with 1 gives $\mathfrak{d}\left(L_{i}\right)=$ $3 / 4+1 / 4 n_{1} n_{2}$.

Now for any integer $i$, the density of $M \cap L_{b}$ in $L_{b}$ varies linearly for $i \leqq b \leqq i+1$, since there are no lattice points on $L_{b}(i<b<i+1)$. We thus obtain the general 
THEOREM 3.6. In the equation $n_{1} X_{2}=n_{2} X_{1}+b$ of the line $L_{b} \subseteq$ $\boldsymbol{R}_{X}$, let $n_{1}$ and $n_{2}$ be nonzero relatively prime integers, and let $\Delta(x)$ be as in Notation 2.4. Then the density $\mathrm{D}\left(L_{b}\right)$ of $M \cap L_{b}$ in $L_{b}$ is given by

$$
\begin{array}{ll}
\mathfrak{D}\left(L_{b}\right)=\frac{3}{4} & \text { if } n_{1} n_{2} \text { is even } ; \\
\mathfrak{D}\left(L_{b}\right)=\frac{3}{4}-\frac{\Delta(b)}{4 n_{1} n_{2}} & \text { if } n_{1} n_{2} \text { is odd } .
\end{array}
$$

So far in this section, we have considered only the case when $L$ has rational slope in $\boldsymbol{R}_{X_{1} X_{2}}$. We next consider the case when $L$ has irrational slope. The main result is

THEOREM 3.7. Let $L$ be any line in $\boldsymbol{R}_{X_{1} X_{2}}=\boldsymbol{R}_{X}$ of irrational slope. Then the density of $M \cap L$ in $L$ is $3 / 4$.

Proof. We use the following convenient notation in this proof: For any line segment $\sigma \subseteq \boldsymbol{R}_{X}$, let $\grave{D}(\sigma)$ denote the density of $M \cap \sigma$ in $\sigma$.

Let the slope of $L$ be $\alpha$. We begin by showing this:

(3.8) For any $\varepsilon>0$, there is a nonzero integer $n$ such that for any $v \in \boldsymbol{R}_{X}$,

$$
\left|\mathfrak{D}(v+[(0,0),(n, \alpha n)))-\frac{3}{4}\right|<\varepsilon .
$$

(3.8.1) follows from the next two inequalities, using the triangle inequality.

(3.9) There are nonzero integers $n, m$ such that for any $v \in \boldsymbol{R}_{X}$,

$$
\begin{gathered}
\left|\mathfrak{D}(v+[(0,0),(n, m)))-\frac{3}{4}\right|<\frac{\varepsilon}{2} ; \\
|\mathfrak{D}(v+[(0,0),(n, m)))-\mathfrak{D}(v+[(0,0),(n, \alpha n)))|<\frac{\varepsilon}{2} .
\end{gathered}
$$

Proof of (3.9.1). For all sufficiently large $n, m$, we have $|1 / n m|<$ $\varepsilon / 2$. From Theorem 3.6 we see that $|\mathfrak{D}([(0,0),(n, m)))-3 / 4|<1 /$ $|n m|(<\varepsilon / 2)$. Also, the segment $[(0,0),(n, m))$ is an "intersection period" for $M \cap\left\{X_{2}=n / m X_{1}\right\}$. This, together with Theorem 3.6 shows that as we translate $[(0,0),(n, m))$ by any $v \in \boldsymbol{R}_{X}$, the oscillation in density never exceeds $1 /|n, m|$; this last is just what (3.9.1) says. 
Proof of (3.9.2). By an easy uniform continuity argument, we see that the left-hand side of (3.9.2) is arbitrarily small for all $|m-\alpha n|$ sufficiently small. That $|m-\alpha n|$ can in fact be made arbitrarily small is guaranteed by [Niven, Theorem 4.3].

Theorem 3.7 now follows at once from (3.8) - just cover $L$ with disjoint copies of the interval $[(0,0),(n, \alpha n))$, and let these intervals be the " $T_{\imath}$ " in Definition 2.1 .

In the foregoing, we have assumed that $a \cdot X$ and $a^{\prime} \cdot X$ were of the simple form $X_{1}$ and $X_{2}$, respectively. We can combine our results and state them in a more general setting this way:

THEOREM 3.10. Let $a=\left(a_{1}, a_{2}\right)$ and $a^{\prime}=\left(a_{1}^{\prime}, a_{9}^{\prime}\right)$ be linearly independent in $\boldsymbol{R}_{X_{1} x_{2}}=\boldsymbol{R}_{X}$. Then any line in $\boldsymbol{R}_{X}$ can be written as

$$
L_{b}: a^{\prime} \cdot X=m(a \cdot X)+b
$$

for some $m \in \boldsymbol{R} \cup\{\infty\}$ and $b \in \boldsymbol{R} . \quad$ (If $m=\infty$, write $a \cdot X=b$.) Let $\mathfrak{D}\left(L_{b}\right)$ denote the density of $\left(V^{*}(a \cdot X) \cup V^{*}\left(a^{\prime} \cdot X\right)\right) \cap L_{b}$ in $L_{j}$. Then:

$$
\begin{aligned}
& \left\{\begin{array}{l}
\mathfrak{D}\left(L_{b}\right)=1\left(b \in A^{1}\right) \\
\mathfrak{D}\left(L_{b}\right)=\frac{1}{2}\left(b \notin A^{1}\right)
\end{array} \quad \text { if } m=0 \text { or } \infty ;\right. \\
& \mathfrak{D}\left(L_{b}\right)=\frac{3}{4}-\frac{4\left(n_{1} b\right)}{4 n_{1} n_{2}} \quad \text { if } m=\frac{n_{2}}{n_{1}} \in \boldsymbol{Q} \backslash\{0\} \quad\left(n_{1}, n_{2}\right. \text { relatively } \\
& \text { prime) and } n_{1} n_{2} \text { is odd; } \\
& \mathfrak{D}\left(L_{b}\right)=\frac{3}{4} \quad \text { for all other } m .
\end{aligned}
$$

4. A density theorem in higher dimensions: Linear case. The density theorems of the last section can be generalized to higher dimensions. In this section we prove one such generalization (Theorem 4.3); this result will be used in $\S 6$, where we consider the general algebraic case. We begin with two definitions.

Definition 4.1. Let $C^{n}$ denote the unit $n$-cube $[0,1) \times \cdots \times$ $[0,1) \subseteq \boldsymbol{R}^{n}$. The subset $\boldsymbol{A}^{n}=\left\{C^{n}+\left(k_{1}, \cdots, k_{n}\right) \mid \sum_{i=1}^{n} k_{i}\right.$ iseven $\left.\left(k_{i} \in \boldsymbol{Z}\right)\right\}$ of $\boldsymbol{R}^{n}$ is called the standard $n$-dimensional checkerboard in $\boldsymbol{R}^{n}$ (or, in view of Definition 5.1, the standard linear alternation on $\left.\boldsymbol{R}^{n}\right)$. For any $\left(k_{1}, \cdots, k_{n}\right)\left(k_{i} \in Z\right), C^{n}+\left(k_{1}, \cdots, k_{n}\right)$ is called a black cube of $\boldsymbol{A}^{n}$ if $\sum_{i=1}^{n} k_{i}$ is even, and a white cube of $\boldsymbol{A}^{n}$ if $\sum_{r=1}^{n} k_{i}$ is odd.

The following is a direct generalization of Definition 2.1. 
DEFINITION 4.2. Let $V \cong \boldsymbol{R}^{n}$ be a real algebraic variety of dimension $r$, and let $V^{\prime}$ be the set of points of $V$ having dimension $r$. For $S \subseteq V$, suppose $S \cap V^{\prime}$ is a countable union of $r$-dimensional regions in $V^{\prime}$. Then $S$ has density $\delta$ in $V$ if for any $\varepsilon>0$, there is a decomposition of $V^{\prime}$ into the disjoint union of countably many uniformly bounded regions $T_{i} \subseteq V^{\prime} \subseteq \boldsymbol{R}^{n}$ so that

$$
\left|\mathfrak{D}-\frac{r-\operatorname{vol}\left(S \cap T_{i}\right)}{r-\operatorname{vol} T_{i}}\right|<\varepsilon
$$

for all but possibly finitely many $T_{i}$.

The next theorem generalizes Theorems 3.6-7.

THEOREM 4.3. Assume the following notation.

Let:

$$
\begin{aligned}
& X \text { denote }\left(X_{1}, \cdots, X_{n}\right) \text {; } \\
& r \leqq \frac{n}{2}(r, a \text { positive integer }) \text {; } \\
& V_{1}^{*}=\text { inverse image of } \boldsymbol{A}^{r} \text { under the natural projection map } \\
& \pi_{1}: \boldsymbol{R}_{X} \longrightarrow \boldsymbol{R}_{X_{1}}, \cdots, X_{r} \text {; } \\
& V_{2}^{*}=\text { inverse image of } \boldsymbol{A}^{r} \text { under the natural projection map } \\
& \pi_{2}: \boldsymbol{R}_{X} \longrightarrow \boldsymbol{R}_{X_{r+1}, \cdots, X_{2}} ; \\
& M=V_{1}^{*} \cup V_{2}^{*} \text {; } \\
& m=\left(m_{1}, \cdots, m_{r}\right) \quad\left(m_{i} \in \boldsymbol{R} \cup\{\infty\}\right) \text {. }
\end{aligned}
$$

Let $V_{b}$ denote the linear $(n-r)$-variety in $\boldsymbol{R}_{X}$ defined by

$$
\left\{\begin{array}{ll}
X_{i+r}=m_{\imath} X_{i}+b_{i} & \left(m_{i} \in \boldsymbol{R}\right) \\
X_{i}=b_{i} & \left(m_{i}=\infty\right)
\end{array}\right\},(i=1, \cdots, r) .
$$

If $m_{i} \in \boldsymbol{Q}_{r}$, write $m_{i}=n_{i 2} / n_{i 1}\left(n_{i 1}, n_{i 2}\right.$ relatively prime). If $m \in \boldsymbol{Q}^{r}$, let $\Pi$ denote $\prod_{i=1}^{r} n_{i 1} \cdot n_{i 2}$, and let $\Delta\left(n_{1} b\right)=\left(\Delta\left(n_{11} b_{1}\right), \cdots, \Delta\left(n_{r 1} b_{r}\right)\right)$. Finally, let $\triangleright\left(V_{b}\right)$ denote the density of $M \cap V_{b}$ in $V_{b}$. Then:

$$
\left\{\begin{array}{l}
\mathfrak{D}\left(V_{b}\right)=1\left(b \in A^{r}\right) \\
\mathfrak{D}\left(V_{b}\right)=\frac{1}{2}\left(b \notin A^{r}\right)
\end{array} \quad \text { if for each } i, m_{i} \text { is either } 0 \text { or } \infty ;\right.
$$

$$
\grave{D}\left(V_{b}\right)=\frac{3}{4}-\frac{\Delta\left(n_{1} b\right)}{4 \Pi} \text { if } m \in \boldsymbol{Q}^{r} \text { and } \Pi \text { is odd ; }
$$

$$
\mathfrak{D}\left(V_{b}\right)=\frac{3}{4} \quad \text { for all other } m \text {. }
$$


Proof. In this first paragraph we give an overall outline of the proof. First, since $M=\left(M \cap \boldsymbol{R}_{X_{1}, \cdots, X_{2 r}}\right) \times \boldsymbol{R}_{X_{2 r+1}, \cdots, X_{n}}$ and $V_{b}=$ $\left(V_{b} \cap \boldsymbol{R}_{X_{1}, \cdots, X_{2 r}}\right) \times \boldsymbol{R}_{X_{2 r+1}, \cdots, X_{n}}$, we may compute $\delta\left(V_{b}\right)$ in (4.3.1-3) by working in $\boldsymbol{R}_{X_{1}, \cdots, X_{2}}$. Therefore, without loss of generality, we assume that $n=2 r$. Next, note that (4.3.1) is immediate from the definitions of $\delta, V_{b}$, and $\boldsymbol{A}^{r}$. We therefore begin with the case when each $m_{i} \in \boldsymbol{Q} \backslash\{0\}$. After that, we consider the case when each $m_{i} \in \boldsymbol{R} \backslash\{0\}$ and at least one $m_{i}$ is irrational. Finally, we complete all possibilities by extending the above to include the case when for at least one but not all $i, m_{i}$ has the value 0 or $\infty$.

We now consider the case when each $m_{i} \in \boldsymbol{Q} \backslash\{0\}$. By renumbering coordinates if necessary, we may assume that $n_{11}, \cdots, n_{r 1}$ are all odd.

We begin by defining two rectangular parallelotopes, $P$ and $P^{\prime}$. $P$ is the image of the unit cube $C^{n}$ under the diagonal map

$$
\left(\begin{array}{ccccc}
n_{11} & & & & \\
& \ddots & & 0 & \\
& & n_{r 1} & & \\
& & n_{12} & \\
& 0 & & \ddots & \\
& & & & n_{r 2}
\end{array}\right)
$$

and $P^{\prime}$ is the image of $C^{n}$ under

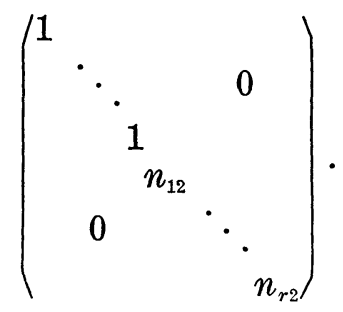

Also, let $v_{0}$ denote $\left(n_{11}, \cdots, n_{2 r}\right)$.

Now in the proof of Theorem 3.6, make these changes:

First, replace the "period" line segment $S=\left[(0,0),\left(2 n_{1}, 2 n_{2}\right)\right)$, which equals $S_{1} \cup S_{2}=\left[(0,0),\left(n_{1}, n_{2}\right)\right) \cup\left[\left(n_{1}, n_{2}\right),\left(2 n_{1}, 2 n_{2}\right)\right)$, by the "period" rectangular parallelotope $\left(V_{0} \cap P\right) \cup\left(v_{0}+V_{0} \cap P\right)$.

Second, replace the old $R_{i}$ by:

$$
R_{i_{1}, \cdots, i_{r}}=\left(i_{1}, \cdots, i_{r}, 0, \cdots, 0\right)+P^{\prime} \quad\left(1 \leqq i_{k} \leqq n_{r}\right) ;
$$

and replace $R_{i}^{\prime}$ by

$$
R_{i_{1}, \cdots, i_{r}}^{\prime}=v_{0}+R_{i_{1}, \cdots, i_{r}} .
$$

Now interchange $M \cap R_{i_{1}, \cdots, i_{r}}$ with $M \cap R_{i_{1}, \cdots, i_{r}}^{\prime}$, for $i_{1}+\cdots+i_{r}$ 
odd. Then in place of $M \cap P$ and $M \cap P^{\prime}$ we now have a black parallelotope and an $n$-dimensional checkerboard parallelotope. To prove our theorem (when each $m_{i} \in \boldsymbol{Q} \backslash\{0\}$ ), we want to show that the density of $V_{b}$ in the checkerboard parallelotope is $1 / 2-\Delta(b) / 2 \Pi$ if $\Pi$ is odd, and $1 / 2$ if $\Pi$ is even.

We do this as follows. First, consider the case $b=0$. An easy induction on $r$ shows that finding the density of $M \cap\left(V_{0} \cap P\right)$ in $V_{0} \cap P$ reduces to counting the black versus the white $n$-cubes in the checkerboard pattern in $P$. If all $n_{i_{2}}$ are odd, there is one more white than black cube, and the density is $1 / 2-1 / 2 \Pi$. If any of the $n_{i_{2}}$ are even, the number of white cubes is the same as the number of black cubes, so the density is $1 / 2$. If $b \in Z^{r}$, then the intersection pattern of $M$ in $V_{b}$ is the same (up to a translation) as above if $\sum_{i=1}^{r} b_{i}$ is even; black and white is everywhere reversed if $\sum_{i=1}^{r} b_{i}$ is odd, and one gets $1 / 2+1 / 2 \Pi$ in place of $1 / 2-1 / 2 \Pi$.

Now, in analogy to the case of $n=2, r=1$ (Theorem 3.6), consider the parallelotope $A$ which is the image of $C^{n}$ under the linear map $T$, where $T$ transforms the canonical basis vectors $\varepsilon_{i}$ this way:

$$
\begin{array}{ll}
\varepsilon_{\imath} \longrightarrow n_{i 1} \varepsilon_{i}+n_{i 2} \varepsilon_{i+r} & (i=1, \cdots, r) \\
\varepsilon_{i} \longrightarrow n_{i 2} \varepsilon_{i} & (i=r+1, \cdots, 2 r) .
\end{array}
$$

There are exactly $\Pi$ (integral) lattice points in $A$, and, for each of the $I I r$-dimensional linear varieties $V_{k_{i}}$ defined by

$$
n_{\imath 1} X_{i+r}=n_{\imath 2} X_{i}+k_{i} \quad\left(k_{i}=0,1, \cdots, n_{i 1} n_{i 2}-1 ; i=1, \cdots, r\right),
$$

there is just one of these lattice points in $A \cap L_{k_{i}}$. (The argument is an easy extension of the one used in Theorem 3.6.) We know the value of the density on each such $V_{k_{i}}$, and the density clearly varies multilinearly off these lattice points. This then proves Theorm 4.3 when each $m_{i} \in \boldsymbol{Q} \backslash\{0\}$.

As for the case when each $m_{i} \in \boldsymbol{R} \backslash\{0\}$ and at least one $m_{i}$ is irrational, the proof is like that of Theorem 3.7, except for routine modifications. Note that in (3.8.1), $v \in \boldsymbol{R}^{2}$ becomes $v \in R^{2 r}$, and " $(n$, $\alpha n)$ " becomes " $\left(n_{1}, \cdots, n_{r}, m_{1} n_{1}, \cdots, m_{r} n_{r}\right)$."

Lastly, we consider the case when for at least one but not all $i$, $m_{i}$ has the value 0 or $\infty$. Suppose without loss of generality that the first $s(1 \leqq s \leqq r-1)$ entries of $\left(m_{1}, \cdots, m_{r}\right)$ are in $R \backslash\{0\}$, and that each of $m_{s+1}, \cdots, m_{r}$ is either 0 or $\infty$. We begin with the case $s=r-1$ and $m_{r}=0$.

Let $H$ denote the hyperplane of $\boldsymbol{R}_{X_{1}, \cdots, X_{2 r}}$ defined by $X_{2 r}=b_{r}$. Let $\pi$ be the natural projection of $H$ onto $\boldsymbol{R}_{X_{r+1}, \cdots, X_{r+s}}$, and $\pi^{\prime}$, the natural projection of $H$ onto $\boldsymbol{R}_{X_{r}}$. Then note: 
(a) The density of the part of $V_{b} \cap M \pi$-lying above anyblack $s$-cube of $\boldsymbol{A}^{s} \leqq \boldsymbol{R}_{x_{r+1}, \cdots, x_{r+s}}$, is 1 .

(b) The intersection pattern of the part of $V_{b} \cap M \pi$-lying above any white $s$-cube of $\boldsymbol{A}^{s} \subseteq \boldsymbol{R}_{X_{r+1}, \cdots, X_{r+s}}$ is periodic in the $X_{r}$-direction. In fact, for any $a \in \boldsymbol{R}$, the part $\pi^{\prime}$-lying above $[a, a+1)$ is, up to a translation, the set-theoretic complement of that $\pi^{\prime}$-lying above $[a+1, a+2)$. Therefore the density of the part of $V_{b} \cap M \pi$-lying above any white $s$-cube $A^{s}$ is $1 / 2$.

Now averaging the above two densities yields $3 / 4$. One can use an analogous argument for $m_{r}=\infty$, replacing $\pi$ and $\pi^{\prime}$ by projections of $H: X_{r}=b_{r}$ onto $\boldsymbol{R}_{X_{1}, \cdots, X_{s}}$ and $\boldsymbol{R}_{2 r}$, respectively. Finally, for any fixed $s$, it is clear that by repeated applications of the above argument, one can increase $r$ from $s+1$ to $s+2$, then to $s+3, \cdots$, thus proving Theorem 4.3 when for at least one but not all $i, m_{i}$ has the value 0 or $\infty$.

This completes the proof of Theorem 4.3.

5. Polynomial alternations; Generalities. Essentially everything we have done so far can be generalized to the polynomial level. In this section we define the notion of "polynomial alternation" and make some first observations about them.

Definition 5.1. Let $X=\left(X_{1}, \cdots, X_{n}\right)$; let $p_{i} \in \boldsymbol{R}[X]$, and let $p=\left(p_{1}, \cdots, p_{r}\right)$. (Then $p$ maps from $\boldsymbol{R}_{X}$ to $\boldsymbol{R}^{r}$.) The inverse image $p^{-1}\left(\boldsymbol{A}^{r}\right) \cong \boldsymbol{R}_{X}$ is called a polynomial alternation on $\boldsymbol{R}_{X}$ (or, often, simply an alternation), and is denoted by $V^{*}(p) \subseteq \boldsymbol{R}_{X}$ (or sometimes by simply $V^{*}$ if $p$ is clear from context).

EXAMPLE 5.2.

(5.2.1). $\quad V^{*}\left(X_{i}\right)(i=1,2)$ describes a set of bands in $\boldsymbol{R}_{X_{1} X_{2}}$ running parallel to $\boldsymbol{R}_{X_{i}}$.

(5.2.2). $\quad V^{*}\left(X_{1}, \cdots, X_{n}\right)$ is the standard $n$-dimensional checkerboard $\boldsymbol{A}^{n} \subseteq \boldsymbol{R}_{X} . \quad V^{*}\left(k X_{1}, \cdots, k X_{n}\right) \quad(k>0)$ is a "finer" checkerboard, in which the edge of each cube is $1 / k$ th as long as in the first case.

(5.2.3). $V^{*}\left(X_{1}^{2}+X_{2}^{2}\right) \subseteq \boldsymbol{R}_{X_{1} X_{2}}$ describes a "Fresnel zone plate" (cf. Figures 2 and 3). (It turns out that the areas of all white and black regions are the same.) $V^{*}\left(X_{1}^{2}+X_{2}^{2}\right) \subseteq \boldsymbol{R}_{X_{1} X_{2} X_{3}}$ could be called "Fresnel cylinders"; $V^{*}\left(X_{1}^{2}+\cdots+X_{n}^{2}\right) \cong \boldsymbol{R}^{n}$ is a set of $n$-dimensional regions bounded by concentric $(n-1)$-spheres; one might describe this alternation as an " $n$-dimensional spherical Fresnel alternation."

Operations on Polynomial Alternations. Polynomial alternations are closed under certain basic operations: 
(5.3.1). Complement of an alternation. With notation as in Definition 5.1 we see that the (set-theoretic) complement $\overline{V^{*}(p)}$ of $V^{*}(p) \subseteq \boldsymbol{R}_{X}$ is an alternation since the complement equals, for instance, $V^{*}\left(p_{1}+1, p_{2}, \cdots, p_{r}\right) \subseteq \boldsymbol{R}_{X}$.

(5.3.2). Addition $\bmod 2$. Let $V_{1}^{*}$ and $V_{2}^{*}$ be two alternations on $\boldsymbol{R}_{X}$. Define $V_{1}^{*}+V_{2}^{*}$ to be $\left(V_{1}^{*} \cap V_{2}^{*}\right) \cup\left(\bar{V}_{1}^{*} \cap \bar{V}_{2}^{*}\right)$. (If one assigns the value 0 to points in $V_{i}^{*}, 1$ to points outside $V_{i}^{*}$, and adds $\bmod 2$, then $V_{1}^{*}+V_{2}^{*}$ is the zero set.)

LEMMA 5.4. Let $V_{1}^{*}$ and $V_{2}^{*}$ be alternations on $\boldsymbol{R}_{X}$. Then $V_{1}^{*}+V_{2}^{*}$ is also an alternation on $\boldsymbol{R}_{X}$.

Proof. Let $V_{1}^{*}=V^{*}\left(p_{1}, \cdots, p_{r}\right)$ and $V_{2}^{*}=V^{*}\left(q_{1}, \cdots, q_{s}\right)$, where $p_{\imath}, q_{j} \in R[X]$. We show that $V_{1}^{*}+V_{2}^{*}=V^{*}\left(p_{1}, \cdots, p_{r}, q_{1}, \cdots, q_{s}\right)$. First, for any $(x) \in \boldsymbol{R}_{x},(x) \in V^{*}\left(p_{1}, \cdots, q_{r}\right)$ iff $\left(p_{1}(x), \cdots, q_{s}(x)\right) \in C^{r+s}+$ $\left(k_{1}, \cdots, k_{r}, k_{1}^{\prime}, \cdots, k_{s}^{\prime}\right) \subseteq R^{r+s}$, where $\sum_{i, j}\left(k_{i}+k_{j}^{\prime}\right)$ is even. (Cf. Definition 4.1.) This can happen iff $\sum_{i} k_{i}$ and $\sum_{j} k_{j}^{\prime}$ are both even or both odd. In either case, $(x) \in V_{1}^{*}+V_{2}^{*}$. Similarly, if just one of $\sum_{i} k_{i}$ and $\sum_{j} k_{j}^{\prime}$ is odd, then $(x) \notin V_{1}^{*}+V_{2}^{*}$.

REMARK 5.5. Algebraic alternations are in general not preserved under either union or intersection. (For instance, neither $V^{*}\left(X_{1}\right) \cup$ $V^{*}\left(X_{2}\right)$ nor $V^{*}\left(X_{1}\right) \cap V^{*}\left(X_{2}\right)$ is an algebraic alternation - the density of the first set is $3 / 4$, and that of the second set is $1 / 4$, both of which contradict Theorem 5.13 .

However, the existence of fringes in the union (and, as it turns out, dually in the intersection) suggests that the union and intersection are trying to act like algebraic alternations. We look at this in the next section.

One can ask for a notion of dimension of polynomial alternations. First, in the complex setting, we know that a variety $V \cong$ $C_{X_{1}, \cdots, X_{n}}$ defined by polynomials $p_{1}, \cdots, p_{r} \in C\left[X_{1}, \cdots, X_{n}\right]$ has dimension $n-r$ provided the $r \times n$ Jacobian matrix $\left(\partial p_{i} / \partial X_{i}\right)$ has rank $r$ at almost every point of $V$. Of course for real varieties, this is far from true. But in the case of polynomial alternations, we are using families of real algebraic varieties, and the nice behavior of the complex case is in large part recaptured. Lemma 5.6 and Theorem 5.7 given next, are basic in this regard.

LEMMA 5.6. Let $p \in \boldsymbol{R}\left[X_{1}, \cdots, X_{n}\right] \backslash \boldsymbol{R}$. Then $p(\boldsymbol{R})$ is either $\boldsymbol{R}$ or a half-infinite interval; and for all but finitely many $b \in p(\boldsymbol{R})$, $p^{-1}(b)$ is a smooth real algebraic variety of pure (real) dimension $n-1$. 
Proof. The first part of theorem is obvious, since a polynomial function on $\boldsymbol{R}$ is either constant or unbounded, and $p(\boldsymbol{R})$ is connected. For the second part, let $b$ be any element of $C$. Then the zero set in $C^{n}$ of $p-b$ is a variety $V_{b}$. By Bertini's theorem, for all but finitely many $b \in C, V_{b}$ is a nonsingular variety of complex dimension $n-1$. Thus for all but finitely many $b \in C$, the Jacobian matrix of $p-b$ at any point $P \in V_{b}$ has rank $n-1$. But $p$ has real coefficients, so for all but finitely many $b \in \boldsymbol{R}$, at any $P \in V_{b} \cap \boldsymbol{R}^{n}$, the Jacobian matrix of $p-b$ is real and has real rank $n-1$. By the real differentiable implicit mapping theorem, the part of $V_{b} \cap \boldsymbol{R}^{n}$ near $P$ is the graph of a $C^{\infty}$-mapping - that is, $V_{b}$ is smooth at $P$.

TheOREM 5.7. Let $p_{1}, \cdots, p_{r} \in \boldsymbol{R}\left[X_{1}, \cdots, X_{n}\right] \backslash \boldsymbol{R}$. If the $r \times n$ Jacobian matrix $J(p)=\left(\partial p_{i} / \partial X_{j}\right)$ has rank $r$, then at almost all points $p \in \boldsymbol{R}^{n}$, the level variety of $\left(p_{1}, \cdots, p_{r}\right)$ in $\boldsymbol{R}^{n}$ which contains $P$, is smooth and has pure real dimension $n-r$.

Proof. The proof is similar to that of Lemma 5.6. (Cf. [Kendig, Chapter II, §3 and IV, §2].

Theorem 5.7 suggests the following definition.

DEFINITION 5.8. A polynomial alternation $V^{*}$ is called regular if $V^{*}=V^{*}\left(p_{1}, \cdots, p_{r}\right) \subseteq \boldsymbol{R}^{n}$ for some $p_{1}, \cdots, p_{r} \in \boldsymbol{R}\left[X_{1}, \cdots, X_{n}\right] \backslash \boldsymbol{R}$, where the $r \times n$ Jacobian matrix $J(p)=\left(\partial p_{i} / \partial X_{j}\right)$ has rank $r$. In this case, $V^{*}$ has dimension $n-r$, and we write $\operatorname{dim} V^{*}=n-r$.

It is easy to check that the above definition of dimension is well-defined - that is, two regular algebraic alternations $V_{1}^{*}$ and $V_{2}^{*}$ in $\boldsymbol{R}^{n}$ having different dimensions, cannot be identical subsets of $\boldsymbol{R}^{n}$.

For any regular alternation $V^{*} \subseteq \boldsymbol{R}^{n}$, if one defines $\operatorname{cod} V^{*}$ to be $n-\operatorname{dim} V^{*}$, then one can prove the following analogue for intersecting algebraic varieties in $\boldsymbol{P}^{n}(\boldsymbol{C})$ :

THEOREM 5.9. Let $V_{1}^{*}, V_{2}^{*}$, and $V_{1}^{*}+V_{2}^{*}$ be regular alternations on $\boldsymbol{R}^{n}$. Then

$$
\operatorname{cod}\left(V_{1}^{*}+V_{2}^{*}\right) \leqq \operatorname{cod} V_{1}^{*}+\operatorname{cod} V_{2}^{*} .
$$

Definition 5.10. If $\operatorname{cod}\left(V_{1}^{*}+V_{2}^{*}\right)=\operatorname{cod} V_{1}^{*}+\operatorname{cod} V_{2}^{*}$, then we say that $V_{1}^{*}$ and $V_{2}^{*}$ add properly.

EXAMPLE 5.11. If $V_{1}^{*}=V_{2}^{*} \subseteq \boldsymbol{R}^{n}(n \geqq 1)$, then $\operatorname{cod}\left(V_{1}^{*}+V_{2}^{*}\right) \varsubsetneqq$ $\operatorname{cod} V_{1}^{*}+\operatorname{cod} V_{2}^{*}$. 
REMARK 5.12. In this paper, "alternation" will henceforth mean "regular algebraic alternation" unless stated otherwise.

We next observe that densities of alternations are exactly $1 / 2$, both "globally" and "locally." Since we do not use these facts (except in Remark 5.5), we only sketch the proofs here. First, at the global level we have:

THEOREM 5.13. Let $V^{*}=V^{*}\left(p_{1}, \cdots, p_{r}\right) \subseteq \boldsymbol{R}^{n}$ be a regular alternation on $\boldsymbol{R}^{n}$, where each $p_{i} \in \boldsymbol{R}\left[X_{1}, \cdots, X_{n}\right] \backslash \boldsymbol{R}$. Then the density of $V^{*}$ in $\boldsymbol{R}^{n}$ is 1/2. (Cf. Definition 4.2.)

Proof. We begin with the case $r=1$ - i.e., $V^{*}=V^{*}\left(p_{1}\right) \subseteq \boldsymbol{R}^{n}$. If there is an $X_{i}$ appearing in $p_{1}$ only as $a X_{i}(a \in R \backslash\{0\})$ and not in any other term, then the level varieties $p^{-1}(n)(n \in \boldsymbol{Z})$ are translates of $p^{-1}(0)$ by integral scalar multiples of some fixed vector, and $V^{*}\left(p_{1}\right)$ obviously has density $1 / 2$.

Therefore (still assuming $r=1$ ), suppose that no $X_{i}$ appears only as $a X_{i}(a \in \boldsymbol{R} \backslash\{0\})$. Consider the graph of the function $Z=$ $p_{1}\left(X_{1}, \cdots, X_{n}\right)$. At any point $\left(x_{1}, \cdots, x_{n}, p\left(x_{1}, \cdots, x_{n}\right)\right)$ of the graph such that the absolute value $\left|p\left(x_{1}, \cdots, x_{n}\right)\right|$ is sufficiently large, the tangent space to the graph is very close to the graph, and the angle between the tangent space and $\boldsymbol{R}_{Z}$ is small. Therefore around $\left(x_{1}, \cdots, x_{n}\right)$, the part of the level sets $p^{-1}(n)(n \in Z)$ are nearly linear, closely spaced, and nearly equally spaced. From Definition 4.2 , it is easy to check that the density of $V^{*}\left(p_{1}\right)$ is $1 / 2$.

Now let $r$ be arbitrary. Then the boundaries of $V^{*}\left(p_{1}\right), \cdots$, $V^{*}\left(p_{r}\right)$ intersect transversally almost everywhere, so that outside some sufficiently large $n$-ball in $\boldsymbol{R}^{n}$, the level sets are almost everywhere locally like cylindrizations of nonsingular linear transformations of $r$-dimensional checkerboards, and again one can check that the density of $V^{*}\left(p_{1}, \cdots, p_{r}\right)$ in $\boldsymbol{R}^{n}$ is $1 / 2$.

Next, at the local level, we can define the following notion of "local density":

Definition 5.14. Let $V^{*}=V^{*}\left(p_{1}, \cdots, p_{r}\right) \cong \boldsymbol{R}^{n}$ be a (regular) polynomial alternation, where $p_{i} \in \boldsymbol{R}\left[X_{1}, \cdots, X_{n}\right] \backslash \boldsymbol{R}$. Let $P$ be a point of $\boldsymbol{R}^{n}=\boldsymbol{R}_{X_{1}, \cdots, X_{n}}$, and let $B(P ; \rho)$ be an $n$-ball in $\boldsymbol{R}^{n}$ centered at $P$ and having radius $\rho$. Then the limit density of $V^{*}$ at $P$ is $D$ provided the following holds; Given any $\varepsilon>0$ there exists a $\delta>0$ such that for any $\rho(\delta \geqq \rho>0)$, there is a positive integer $k_{\rho}$ such that for any integer $k \geqq k_{r}$,

$$
\left|\mathfrak{D}-\frac{n \text {-volume }\left[V^{*}\left(k p_{1}, \cdots, k p_{r}\right) \cap B(P ; \rho)\right]}{n \text {-volume }(B(P ; \rho))}\right|<\varepsilon .
$$


One may check that $D$ in the above definition is actually welldefined. Using this definition, one can then prove that the limit density of any alternation on $\boldsymbol{R}^{n}$, is $1 / 2$ at each point of $\boldsymbol{R}^{n}$. Since we do not use this result, we leave the proof to the reader.

6. Density theorem for polynomial alternations. In this section we extend the main density result proved in the linear case (Theorem 4.3) to the union of two arbitrary polynomial alternations (Theorem 6.2).

We begin with the following definition.

DeFinition 6.1. Let

(6.1.1) $p=\left(p_{1}, \cdots, p_{r}\right)$ and $q=\left(q_{1}, \cdots, q_{r}\right)\left(p_{i}, q_{j} \in \boldsymbol{R}\left[X_{1}, \cdots, X_{n}\right] \backslash \boldsymbol{R}\right)$,

let $m, b, \Delta\left(n_{1} b\right)$ and $\Pi$ be as in Theorem 4.3, and let $V_{b}$ denote the variety in $\boldsymbol{R}^{n}$ defined by the equations

$$
\left\{\begin{array}{l}
q_{i}=m_{i} p_{i}+b_{i} \\
p^{i}=b_{i}
\end{array}\right\} \begin{aligned}
& \left(m_{i} \in \boldsymbol{R}\right) \\
& \left(m_{i}=\infty\right)
\end{aligned} \quad(1=1,2, \cdots, r) .
$$

Then $\left\{V_{b} \mid b \in \boldsymbol{R}^{r}\right\}$ is called the $m$ th order fringe family of $V^{*}(p) \cup$ $V^{*}(q)$. For convenience, $V^{*}(p) \cup V^{*}(q)$ is sometimes denoted by $M(p, q)$, or simply by $M$ ( $M$ for "moiré").

In analogy to Theorem 4.3, we would like to prove the following result:

THEOREM 6.2. Let $p, q$ be as in (6.1.1), and suppose that $V^{*}(p) \subseteq$ $\boldsymbol{R}^{n}$ and $V^{*}(q) \subseteq \boldsymbol{R}^{n}$ add properly (cf. Definition 5.10). Then with notation as immediately above, we have

$$
\left\{\begin{array}{l}
\mathfrak{D}\left(V_{b}\right)=1 \\
\mathfrak{D}\left(V_{b}\right)=\frac{1}{2}
\end{array}\right.
$$

if for each $i, m_{i}$ is either 0 or $\infty$;

$$
\mathfrak{D}\left(V_{b}\right)=\frac{3}{4}-\frac{\Delta\left(n_{1} b\right)}{4 \Pi} \text { if } m \in \boldsymbol{Q}^{r} \text { and } \Pi \text { is odd; }
$$

$$
\mathfrak{D}\left(V_{b}\right)=\frac{3}{4} \quad \text { for all other } m .
$$

The problem is to define " $\mathrm{D}$ " in (6.2.1-3) to make Theorem 6.2 true. For bounded varieties $V_{b} \subseteq \boldsymbol{R}^{n}$ (or more generally for varieties $V_{b}$ of finite $(n-r)$-dimensional volume), the ordinary "ratio" definition of density, namely $\operatorname{vol}\left(M \cap v_{b}\right) / \operatorname{vol}\left(V_{b}\right)$, does not in general work here. As an example, consider in $\boldsymbol{R}_{X_{1} X_{2}} V^{*}\left(X_{1}^{2}+X_{2}^{2}\right)$ and 
$V^{*}\left(\varepsilon X_{1}\right)$, where $\varepsilon>0$ is very small. Then for small $b$, the above ratio definition of density yields results which are quite different from (6.2.1-3), as a rough sketch will show.

One can take the point of view that the finite "width" of the alternations' bands acts as a kind of "graininess" which introduces error when using the ratio definition. However, as we shall see in a moment, one can "refine" the moire so that as we make higher and higher refinements, the ordinary ratio definition approaches at each point of $V_{b}$, a nice limit density which makes Theorem 6.2 valid. It is interesting to notice that for larger and larger $|b|=$ $\sqrt{b_{1}^{2}+\cdots+b_{2}^{2}}$ ( which means that the distance from $V_{b}$ to $(0)$ gets larger and larger), the $V_{b}$ 's become locally more linear, the bands become finer and more closely spaced, and the ordinary ratio density yields results which, for sufficiently large $|b|$, become arbitrarily close to the density making Theorem 6.2 true.

Definition 6.3. Let $V^{*}(p) \subseteq \boldsymbol{R}^{n}$ and $V^{*}(q) \subseteq \boldsymbol{R}^{n}$ be two properly-adding polynomial alternations of codimension $r(p, q$ are as in (6.1.1)). Let $I=$ identity $r \times r$ matrix, let $k$ be a positive integer, and let $\boldsymbol{A}_{k}^{r}$ be the image of $\boldsymbol{A}^{r}$ under the linear contraction $(1 / k) I$. For any such $k$, and for $m=\left(m_{1}, \cdots, m_{r}\right) \in \boldsymbol{R}^{r}$ let the ordered pair of functions $\left(s_{i}, t_{i}\right)$ be defined by

$$
\left(s_{i}, t_{i}\right)= \begin{cases}\left(p_{i}, m_{i} p_{i}+\frac{1}{k}\left(q_{i}-m_{i} p_{i}\right)\right) & \text { if } m_{i} \in \boldsymbol{R}, \\ \left(\frac{p_{i}}{k}, q_{i}\right) & \text { if } m_{i}=\infty .\end{cases}
$$

Denote $\left(s_{1}, \cdots, s_{r}\right)$ by $s$, and $\left(t_{1}, \cdots, t_{r}\right)$ by $t$. Then $s^{-1}\left(\boldsymbol{A}_{k}^{r}\right) \cup t^{-1}\left(\boldsymbol{A}_{k}^{r}\right)$ is called the k-refinement of the moire $M(p, q)$ along the mth order fringe family, and is denoted by $M_{k}(p, q ; m)$.

EXAMPLE 6.4. Figure 5 represents an unrefined moiré, $M\left(X_{1}\right.$, $\left.X_{2}\right) \subseteq \boldsymbol{R}_{X_{1} X_{2}}$; fringe directions for $m=1$ and $m=1 / 3$ are indicated. In Figure 6, the moiré has been refined along the 1st-order fringe family, with $k=3$. In Figure $7, M\left(X_{1}, X_{2}\right)$ has been refined along the $1 / 3$ order fringe family, again with $k=3$. Note that for fixed $m$, the "refining" or "compressing" is done in the direction of the fringes of the $m$ th order fringe family. The larger $k$ is, the greater the compressing; the spacing between the fringes themselves remains unchanged.

REMARK 6.5. The form of (6.3.1) in Definition 6.3 was actually chosen for a practical application - proving Theorem 6.2, next. 


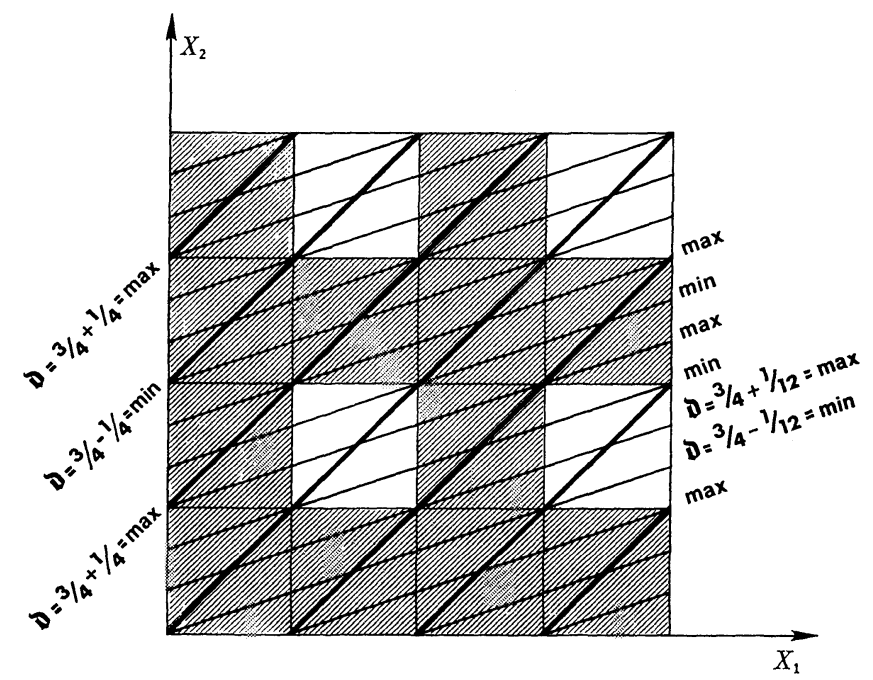

Figure 5

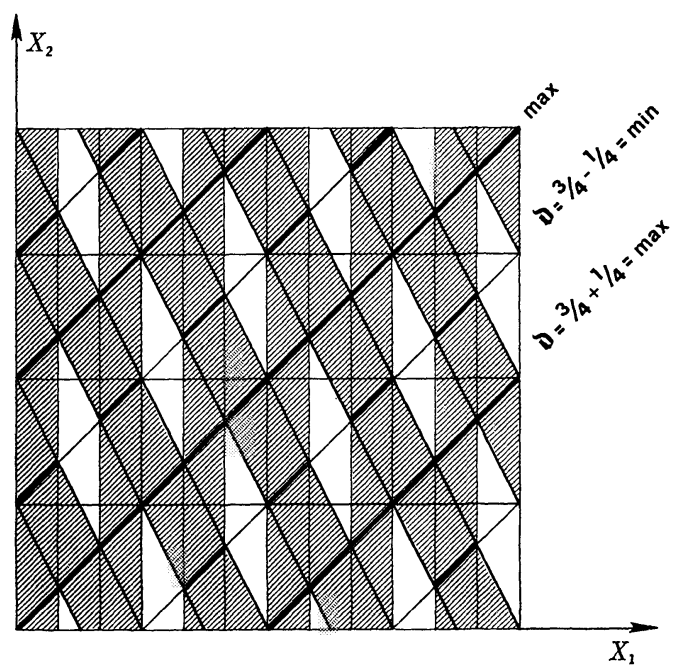

Figure 6

However, one can express the essential idea in Definition 6.3 more symmetrically as follows. Let $\boldsymbol{R}^{*}=\boldsymbol{R} \backslash\{0\}$. Then define

$$
\left(s_{i}, t_{i}\right)=\left\{\begin{array}{l}
\left(p_{i}, m_{i} p_{i}+\frac{1}{k}\left(q_{i}-m_{i} p_{i}\right)\right) \text { if } m_{i} \in R^{*} \cup\{0\}, \\
\left(\frac{q_{i}}{m_{i}}+\frac{1}{k}\left(p_{i}-\frac{q_{i}}{m_{i}}\right), q_{i}\right) \text { if } m_{i} \in R^{*} \cup\{\infty\} .
\end{array}\right.
$$

This agrees with (6.3.1) when $m_{i}=0$ or $\infty$, namely, $\left(p_{i}, q_{i} / k\right)$ if $m_{i}=0$ and $\left(p_{i} / k, q_{i}\right)$ if $m_{i}=\infty$. For $m_{i} \in R^{*},\left(s_{i}, t_{i}\right)$ is not welldefined, but the two densities induced on " $V_{b}$ " in Theorem 6.2 can easily be checked to be the same. For convenience, in (6.3.1) we 


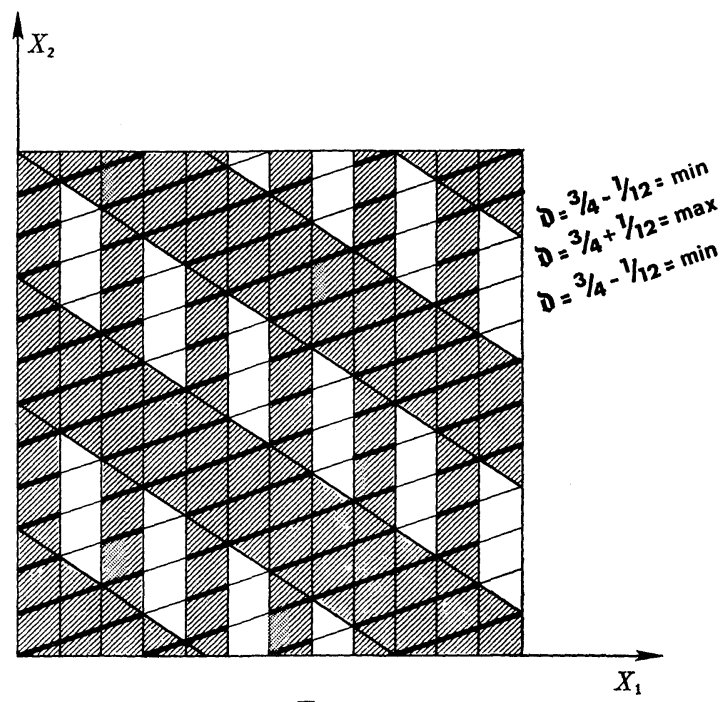

FIgURE 7

have simply chosen the first one for $m_{i} \in \boldsymbol{R}^{*}$.

We next define a local density $D_{P}$ leading to a density on the $V_{b}$ of Theorem 6.2 which will make that theorem true.

DeFinition 6.6. Let $V^{*}(p)$ and $V^{*}(q)$ be properly-adding alternations of codimension $r$ in $\boldsymbol{R}^{n}\left(p, q\right.$ as in (6.1.1)), and let $P \in \boldsymbol{R}^{n}$. Then $M(p, q)$ has limit density $\grave{D}_{P}$ at $P$, with respect to the $m$ th fringe family, if the following holds: For every $\varepsilon>0$, there is a $\delta>0$ such that for any $\rho(\delta>\rho>0)$, there is a positive integer $k_{\rho}$ such that for any integer $k>k_{\rho}$,

$$
\left|\grave{D}_{P}-\frac{n \text {-volume }\left(M_{k}(p, q ; m) \cap B(P, \rho)\right)}{n \text {-volume }(B(P, \rho))}\right|<\varepsilon .
$$

Proof of Theorem 6.2. Let $U_{P}$ be an $\boldsymbol{R}^{n}$-open neighborhood about $P$. We want to estimate, for small $U_{P}$ and sufficiently large $k$, the density in $V_{b} \cap U_{P}$ of

$$
V_{b} \cap\left[s^{-1}\left(\boldsymbol{A}_{k}^{r}\right) \cup t^{-1}\left(\boldsymbol{A}_{k}^{r}\right)\right] .
$$

For a fixed $b$, define

$$
\left(u_{i}, v_{\imath}\right)= \begin{cases}\left(p_{i}, \frac{b_{i}}{k}\right) & \text { if } m_{i}=0, \\ \left(p_{i}, m_{i} p_{i}+\frac{b_{i}}{k}\right) & \text { if } m_{i} \in \boldsymbol{R}^{*}, \\ \left(\frac{b_{i}}{k}, q_{i}\right) & \text { if } m_{i}=\infty ;\end{cases}
$$


and define $u=\left(u_{1}, \cdots, u_{r}\right)$ and $v=\left(v_{1}, \cdots, v_{r}\right)$. Then for any fixed $b,(6.7)$ is easily seen to be the same as

$$
V_{b} \cap\left[u^{-1}\left(\boldsymbol{A}_{k}^{r}\right) \cup v^{-1}\left(\boldsymbol{A}_{k}^{r}\right)\right] .
$$

Thus we want to estimate the density in $V_{b} \cap U_{P}$ of (6.9).

To do this, first let $b \in \boldsymbol{R}^{r}$ be such that the $(\boldsymbol{r} \times n)$-Jacobian matrices $J(p)=\left(\partial p_{i} / \partial X_{i}\right)$ and $J(q)=\left(\partial q_{i} / \partial X_{j}\right)$ each have rank $r$. at almost every point $P \in V_{b}$. (This happens for almost every $b \in \boldsymbol{R}^{r}$.) At any such $P$ where $J(p)$ and $J(q)$ have rank $r$, let $p_{i}^{*}$ (resp. $q_{i}^{*}$ ) denote the initial parts of the expansion of $p_{i}$ (resp. $q_{i}$ ) about $P$; let $\left(u_{i}^{*}, v_{i}^{*}\right)$ be (6.8) with $p_{i}^{*}$ (resp. $q_{i}^{*}$ ) in place of $p_{i}$ (resp. $q_{i}$ ); and let $u^{*}=\left(u_{1}^{*}, \cdots, u_{r}^{*}\right), v^{*}=\left(v_{1}^{*}, \cdots, v_{r}^{*}\right)$. Then in $V_{b} \cap U_{P}, V_{b} \cap M(p$, $q ; m)$ is well-approximated by $V_{b} \cap\left[u^{*-1}\left(\boldsymbol{A}_{k}^{r}\right) \cup v^{*-1}\left(\boldsymbol{A}_{k}^{r}\right)\right]$. Since $J(p)$ and $J(q)$ have maximal rank at $P$, the initial parts are of degree 1; thus for $U_{P}$ sufficiently small and $k$ sufficiently large, the density can be made arbitrarily close to that in the linear case, given in Theorem 4.3. This means that $\mathfrak{D}_{P}$ has a common value on all such points $P$ of $V_{b}$; therefore assigning $V_{b}$ density $\mathfrak{D}=\mathfrak{D}_{P}$ then makes Theorem 6.2 valid in this case.

The density function is now defined at almost all points of $\boldsymbol{R}^{n}$. From the simple form of the density function at these points, we see that it can be extended to a continuous function on $\boldsymbol{R}^{n}$, and that the density thus defined agrees at each point with the density in Definition 6.6. Thus (6.2.1-3) all hold in this case too, and Theorem 6.2 is proved.

REMARK 6.10. Throughout this paper we have always taken the moire to be the union of alternations, which corresponds to the usual physical setup. However, there is a "dual" theory, in which one uses the intersection of alternations. Everything we have done for moirés can be recast in this new setting; essentially, this follows from the duality $V_{1}^{*} \cup V_{2}^{*} \rightarrow \overline{V_{1}^{*} \cup V_{2}^{*}}=\bar{V}_{1}^{*} \cap \bar{V}_{2}^{*}$. In Theorem 6.2, for instance, $3 / 4$ is everywhere replaced by $1-3 / 4=1 / 4$, and $3 / 4-$ $\Delta\left(n_{1} b\right) / 4 \Pi$ becomes $1 / 4+\Delta\left(n_{1} b\right) / 4 \Pi$.

7. Further questions. We have hardly scratched the surface in a study of moirés. There are several directions in which the theory can naturally be continued. For example, one can try to extend everything to the complex setting; in this case, an algebraic alternation is the inverse image of $\boldsymbol{A}^{2 n} \cong \boldsymbol{C}^{n}$ under a polynomial mapping. Since every irreducible algebraic variety in $\boldsymbol{C}^{n}$ of dimension $r>0$ has infinite (real) $2 r$-volume, it appears that one can obtain a density formula corresponding to Theorem 6.2 without using refinements, but instead, using an ordinary density like that in 
Definition 4.2. One does not, however, get a good local density this way.

In another direction, one can ask for extensions to the projective setting $\boldsymbol{P}^{n}(\boldsymbol{R})$ or $\boldsymbol{P}^{n}(\boldsymbol{C})$. Here we are essentially generalizing polynomials to rational functions. This leads in a natural way to points (or varieties) of indeterminate density, something like indeterminacy points of elements in a variety's function field.

Also, just as one can extend much of algebraic geometry to its "twin brother" analytic geometry, one can likewise try to extend a study of moirés to the analytic level.

\section{REFERENCES}

1. K. Kendig, Elementary Algebraic Geometry, New York, Springer-Verlag, 1977.

2. I. Niven, Irrational Numbers, Carus Monograph No. 11, New York, John Wiley. and Sons, Inc., 1956.

Received May 8, 1979.

Cleveland State University

Cleveland, OH 44115 



\section{PACIFIC JOURNAL OF MATHEMATICS}

\section{EDITORS}

DONALD BABBITT (Managing Editor)

University of California

Los Angeles, CA 90024

HUGo RossI

University of Utah

Salt Lake City, UT 84112

C. C. MOORE and ANDREW OGG

University of California

Berkeley, CA 94720
J. DugundJI

Department of Mathematics

University of Southern California

Los Angeles, CA 90007

R. FinN and J. Milgram

Stanford University

Stanford, CA 94305

ASSOCIATE EDITORS
E. F. BECKENBACH
B. H. NeUmanN
F. WOLF
K. YoSHIDA

\section{SUPPORTING INSTITUTIONS}

UNIVERSITY OF BRITISH COLUMBIA

CALIFORNIA INSTITUTE OF TECHNOLOGY

UNIVERSITY OF CALIFORNIA

MONTANA STATE UNIVERSITY

UNIVERSITY OF NEVADA, RENO

NEW MEXICO STATE UNIVERSITY

OREGON STATE UNIVERSITY

UNIVERSITY OF OREGON
UNIVERSITY OF SOUTHERN CALIFORNIA

STANFORD UNIVERSITY

UNIVERSITY OF HAWAII

UNIVERSITY OF TOKYO

UNIVERSITY OF UTAH

WASHINGTON STATE UNIVERSITY

UNIVERSITY OF WASHINGTON

The Supporting Institutions listed above contribute to the cost of publication of this Journal, but they are not owners or publishers and have no responsibility for its content or policies.

Mathematical papers intended for publication in the Pacific Journal of Mathematics should be in typed form or offset-reproduced, (not dittoed), double spaced with large margins. Please do not use built up fractions in the text of the manuscript. However, you may use them in the displayed equations. Underline Greek letters in red, German in green, and script in blue. The first paragraph or two must be capable of being used separately as a synopsis of the entire paper. Please propose a heading for the odd numbered pages of less than 35 characters. Manuscripts, in triplicate, may be sent to any one of the editors. Please classify according to the scheme of Math. Reviews, Index to Vol. 39. Supply name and address of author to whom proofs should be sent. All other communications should be addressed to the managing editor, or Elaine Barth, University of California, Los Angeles, California, 90024.

50 reprints to each author are provided free for each article, only if page charges have been substantially paid. Additional copies may be obtained at cost in multiples of 50 .

The Pacific Journal of Mathematics is issued monthly as of January 1966. Regular subscription rate: $\$ 84.00$ a year (6 Vols., 12 issues). Special rato: $\$ 42.00$ a year to individual members of supporting institutions.

Subscriptions, orders for numbers issued in the last three calendar years, and changes of address shoud be sent to Pacific Journal of Mathematics, P.O. Box 969, Carmel Valley, CA 93924, U.S.A Old back numbers obtainable from Kraus Periodicals Co., Route 100, Millwood, NY 10546.

PUBLISHED BY PACIFIC JOURNAL OF MATHEMATICS, A NON-PROFIT CORPORATION

Printed at Kokusai Bunken Insatsusha (International Academic Printing Co., Ltd.). 8-8, 3-chome, Takadanobaba, Shinjuku-ku, Tokyo 160, Japan.

Copyright (C) 1980 by Pacific Jounal of Mathematics Manufactured and first issued in Japan 


\section{Pacific Journal of Mathematics \\ Vol. 89, No. $2 \quad$ June, 1980}

Frank Hayne Beatrous, Jr. and R. Michael Range, On holomorphic

approximation in weakly pseudoconvex domains................. 249

Lawrence Victor Berman, Quadratic forms and power series fields ...... 257

John Bligh Conway and Wacław Szymański, Singly generated antisymmetric operator algebras ....................... 269

Patrick C. Endicott and J. Wolfgang Smith, A homology spectral sequence for submersions . . . .................................

Sushil Jajodia, Homotopy classification of lens spaces for one-relator groups with torsion ................................ 301

Herbert Meyer Kamowitz, Compact endomorphisms of Banach

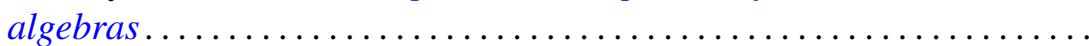

Keith Milo Kendig, Moiré phenomena in algebraic geometry: polynomial

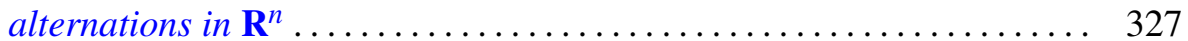

Cecelia Laurie, Invariant subspace lattices and compact operators....... 351

Ronald Leslie Lipsman, Restrictions of principal series to a real form . . . . . 367

Douglas C. McMahon and Louis Jack Nachman, An intrinsic

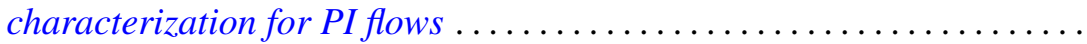

Norman R. Reilly, Modular sublattices of the lattice of varieties of inverse semigroups .................................... 405

Jeffrey Arthur Rosoff, Effective divisor classes and blowings-up of $\mathbf{P}^{2}$ 419

Zalman Rubinstein, Solution of the middle coefficient problem for certain

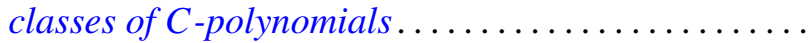

Alladi Sitaram, An analogue of the Wiener-Tauberian theorem for spherical transforms on semisimple Lie groups ................

Hal Leslie Smith, A note on disconjugacy for second order systems ...

J. Wolfgang Smith, Fiber homology and orientability of maps ...

Audrey Anne Terras, Integral formulas and integral tests for series of positive matrices. 